\title{
Evidence of Molecular Fragmentation inside the Charged Droplets Produced by Electrospray Process
}

\author{
Shibdas Banerjee, ${ }^{1}$ Halan Prakash, ${ }^{2}$ Shyamalava Mazumdar ${ }^{1}$ \\ ${ }^{1}$ Department of Chemical Sciences, Tata Institute of Fundamental Research, Homi Bhabha Road, Mumbai 400005, India \\ ${ }^{2}$ Department of Chemistry, Birla Institute of Technology and Science, Pilani, Goa Campus, Goa 403726, India
}

\begin{abstract}
The behavior of the analyte molecules inside the neutral core of the charged droplet produced by the electrospray (ES) process is not unambiguously known to date. We have identified interesting molecular transformations of two suitably chosen analytes inside the ES droplets. The highly stable $\mathrm{Ni}$ (II) complex of 1,8-dimethyl-1,3,6,8,10,13-hexaazacyclotetradecane (1) that consists of a positive charge at the metal center, and the allyl pendant armed tertiary amine containing macrocycle 3,4,5:12,13,14-dipyridine-2,6,11,15-tetramethyl-1,7,10,16-tetraallyl1,4,7,10,13,16-hexaazacyclooctadeca-3,13-diene $\left(\mathbf{M}_{\mathbf{4 p}}\right)$ have been studied by ESI mass spectrometry as the model analytes. We have shown that these two molecules are not representatively transferred from solution to gas phase by ESI; rather, they undergo fragmentation inside the charged droplets. The results indicated that a charged analyte such as 1 was possibly unstable inside the neutral core of the ES droplet and undergoes fragmentation due to the Coulombic repulsion imparted by the surface protons. Brownian motion of the neutral analyte such as $\mathbf{M}_{\mathbf{4 p}}$ inside the droplet, on the other hand, may lead to proton attachment on interaction with the charged surface causing destabilization that leads to fragmentation of $\mathbf{M}_{\mathbf{4 p}}$ and release of resonance stabilized allyl cations from the core of the droplet. Detailed solvent dependence and collision-induced dissociation (CID) studies provided compelling evidences that the fragmentation of the analytes indeed occurs inside the charged ES droplets. A viable model of molecular transformations inside the ES droplet was proposed based on these results to rationalize the behavior of the analyte molecules inside the charged ES droplets.
\end{abstract}

Key words: ESI-MS, Fragmentation, Charged droplet, Coulomb repulsion, Nickel complex, Pendant armed macrocycle

\section{Introduction}

C lectrospray ionization mass spectrometry (ESI-MS) [1$\mathrm{C}_{4}$ ] has evolved into one of the most versatile and widely

Electronic supplementary material The online version of this article (doi:10.1007/s13361-011-0188-7) contains supplementary material, which is available to authorized users.

Correspondence to: Shyamalava Mazumdar; e-mail: shyamal@tifr.res.in; URL: http://www.tifr.res.in/ shyamal/ used analytical technique to study weak noncovalent interactions like intermolecular hydrogen bonding [5-7], protein-protein complexation [8], enzyme-substrate interaction [9] etc. since ESI can representatively transfer those interactions from solution to the gas phase.

Although different mechanisms have been proposed for the formation of gas-phase ions by ESI method [10-16], several aspects of the ionization are still not fully understood. One such issue is the conformational and other subtle chemical properties of the analyte during the transfer from 
solution to gas phase. Some recent works [17, 18] suggested dramatic structural alteration of the proteins after electrospray ionization leading to formation of different conformations [18] of the macromolecules in the gaseous phase. However, the exact reason of the existence of different conformations of the same molecule in the gas phase (unlike in the solvent) is not known.

It is argued that the charges on an ES droplet are distributed over its surface with equidistant spacing and they are locked into this pattern by the forces of Coulomb repulsion [19], and the bulk or core of the liquid droplet essentially remains free of charge to minimize the potential energy of the droplet [20]. Therefore, the neutral solute molecules residing at the core of the droplet were assumed to remain unaffected by the charged environment at the droplet surface. The ESI-MS studies on this aspect have mainly been directed to the biologically important macromolecules like proteins, peptides, and nucleic acids in the last few decades. The charged jet-breakup and subsequent Coulombic explosion of the ES droplets encapsulating the proteins have earlier been assumed to cause repacking of the supramolecular assemblies, forcing them to hold a minimal volume $[21,22]$. However, to the best of our knowledge, there is no direct experimental evidence to support this assumption. The molecular behavior inside the charged ES droplets still remains far from understood.

As discussed above, the charges (protons) reside on the surface of the electrospray droplets and the bulk or core of the droplet remains charge free in order to minimize the potential energy of the droplet [19]. This leads to the hypothesis that if we put a molecule with certain positive charge, which is not contributed by protons, inside the charged droplet, that molecule might be destabilized by huge Coulomb force of repulsion imparted by the surface protons on the droplets. To test this, we have studied two specifically designed molecules $\left(\mathbf{1}\right.$ and $\mathbf{M}_{\mathbf{4 p}}$, Figure 1) by ESI-MS. Molecule $\mathbf{1}$ is a very stable cationic (di-positive) chelate complex, $\mathrm{Ni}(\mathrm{II})$ complex of 1,8dimethyl-1,3,6,8,10,13-hexaazacyclotetradecane (1, Figure 1) $[23,24]$. This square planar complex is highly stable in the acidic solution, and the charge is contributed solely by the central metal ion $\left(\mathrm{Ni}^{2+}\right)[23,24]$. The counter anion of $\mathbf{1}$ is $\mathrm{ClO}_{4}^{-}$, which has very weak interaction with the complex cation in the solution owing to its stable $\pi$-delocalization [25]. Molecule $\mathbf{M}_{\mathbf{4 p}}$ is an allyl pendant armed macrocyclic molecule 3,4,5:12,13,14-dipyridine-2,6,11,15-tetramethyl-1,7,10,16-tetraallyl-1,4,7,10,13,16-hexaazacyclooctadeca-3,13-diene ( $\mathbf{M}_{4 \mathbf{p}}$, Figure 1). These molecules ( $\mathbf{1}$ and $\mathbf{M}_{\mathbf{4 p}}$, Figure 1) could undergo fragmentation inside the charged droplet due to the Coulomb repulsion by the surface protons on the droplets. The results of the ESI-MS studies on these two compounds provided clear evidence that the analyte molecules undergo transformations or change of their structure or even the fragmentation inside the core of the charged droplets depending on their chemical nature.
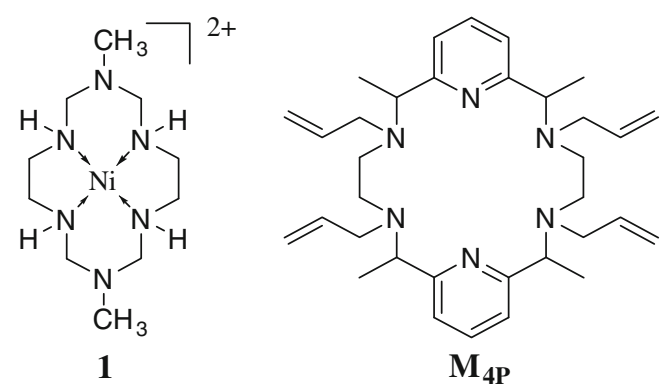

Figure 1. Structure of the Complex 1 [Ni(II) complex of 1,8dimethyl-1,3,6,8,10,13-hexaazacyclotetradecane] and the macrocycle $\mathbf{M}_{\mathbf{4 p}}[3,4,5: 12,13,14$-dipyridine-2,6,11,15- tetramethyl-1,7,10,16-tetraallyl-1,4,7,10,13,16-hexaazacyclooctadeca-4,13-diene] (subscript $4 p$ represents four pendant arms)

\section{Experimental}

\section{Materials}

Nickel(II) chloride hexahydrate, 99\% ethylenediamine, 36\% formaldehyde, $40 \%$ methylamine, perchloric acid, 2,6diacetylpyridine, barium chloride dihydrate, sodium borohydride, anhydrous sodium sulphate, anhydrous potassium carbonate, sodium hydroxide and 3-nitrobenzyl alcohol were provided by Sigma-Aldrich Co. Ltd. AR grade methanol, chloroform, hydrobromic acid were purchased from S. D. Fite Chemicals Ltd. Mumbai, India. Allylbromide was obtained from M/s. Loba Chemie (P) Ltd. Mumbai, India. HPLC grade acetic acid and methanol were purchased from Spectrochem Pvt. Ltd. India. All chemicals were used without further purification.

\section{Synthesis of Ni(II) Complex of 1,8-dimethyl- 1,3,6,8,10,13-hexaazacyclotetradecane (1)}

The Complex 1 was synthesized by the template condensation of ethylenediamine, formaldehyde and methylamine in presence of $\mathrm{Ni}^{2+}$ as the templating cation as reported earlier $[23,26]$.

\section{Synthesis of Pendant Armed Macrocycle}

3,4,5:12,13,14-dipyridine-2,6,11,15-tetramethyl1,7,10,16-tetraallyl-1,4,7,10,13,16hexaazacyclooctadeca-3,13-diene ( $\left.\boldsymbol{M}_{4 p}\right)$

Macrocycle $\mathbf{M}_{4 \mathbf{p}}$ containing four pendant arms was synthesized using the earlier reported method [27] for analogous compounds. The precursor macrocycle 3,4,5:12,13,14-dipyridine-2,6,11,15-tetramethyl-1,4,7,10,13,16-hexaazacyclooctadeca-3,13-diene, synthesized by reduction of the Schiff base formed on reaction of 2,6 diacetyl pyridine and ethylene diamine [27]. This was treated with allylbromide to prepare the macrocycle $\mathbf{M}_{\mathbf{4 p}}$ (isolated yield: $19 \%$ ). The macrocycle $\mathbf{M}_{\mathbf{4}}$ is sparingly soluble in methanol and soluble in dimethylformamide (DMF) but insoluble in all other common organic solvents. M.P: $>250{ }^{\circ} \mathrm{C}$. Elemental 
analysis: observed $75.45 \% \mathrm{C}, 9.30 \% \mathrm{H}$, and $15.25 \% \mathrm{~N}$ against calculated: $75.23 \% \mathrm{C}, 9.28 \% \mathrm{H}$, and $15.48 \% \mathrm{~N}$. ${ }^{1} \mathrm{H}$

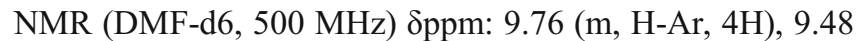
(m, H-Ar, 2H), 5.6-5.2 (m, aliphatic $-\mathrm{CH}_{2}-\mathrm{N},-\mathrm{CH}=,-\mathrm{CH}-$ $\mathrm{N}, 24 \mathrm{H}), 4.47$ and $4.64\left(\mathrm{~m},=\mathrm{CH}_{2}, 8 \mathrm{H}\right), 3.5\left(\mathrm{~m},-\mathrm{CH}_{3}, 12 \mathrm{H}\right)$. FT-IR $\left(\mathrm{KBr}, \mathrm{cm}^{-1}\right)$ : 3052 (aromatic C-H str.), 2986 (asymmetric - $\mathrm{CH} 3$ str.), 2802, 2730 (asymmetric-CH2str.), 1632 (alkene str.), 1599-1446 (aromatic $\mathrm{C}=\mathrm{N}, \mathrm{C}=\mathrm{C}$ str.), 1385 (in plane $\mathrm{C}-\mathrm{H}$ bending of alkene), 1167 (C-N str. in t-amine), 1082, 1022 (in plane aromatic ring $\mathrm{C}-\mathrm{H}$ bending), 823/754/674 (out plane bending of aromatic ring $\mathrm{C}-\mathrm{H}$ ). The spectroscopic characterization of the macrocycle $\mathbf{M}_{4 \mathbf{p}}$ confirmed the presence of four allyl pendant arms attached to the hexaaza macrocycle.

\section{Mass Spectrometry}

Electrospray ionization mass spectrometry (ESI-MS) and collision induced dissociation (ESI-MS/CID) studies were carried out using a Thermo Finnigan LCQ Deca Electrospray quadrupole ion trap mass spectrometer [5, 28-30]. All the experiments were done under identical conditions unless otherwise stated. The flow rate of the analyte solution of concentration $\sim 10 \mu \mathrm{M}$ was maintained at $5 \mu \mathrm{L} / \mathrm{min}$, and the solutions were directly injected through the ion source kept in positive ion mode [31]. Nitrogen was used as the sheath and auxiliary gas. The ion source conditions were: sheath gas flow rate $\sim 7.5 \mathrm{~L} / \mathrm{min}$, with no auxiliary gas flow. Capillary temperature (for desolvation) was maintained at approximately $200{ }^{\circ} \mathrm{C}$ and capillary voltage was kept at $15 \mathrm{~V}$. The ion-spray voltage and tube lens offset were maintained at $+4.5 \mathrm{kV}$ and $-7 \mathrm{~V}$, respectively. Low-energy CID product ion spectra were acquired using an isolation width of $6 \mathrm{~m} / z$ (to select all isotopically distributed peaks of the same species/all neighboring isotopic peaks) for $\mathbf{1}$. The isolation width was $2 \mathrm{~m} / \mathrm{z}$ for $\mathbf{M}_{\mathbf{4 p}}$. The activation Q (related to the parameter $\mathrm{q}_{\mathrm{z}}$ in the Mathieu equation [32] for the precursor ions) of 0.250 with an activation time $30 \mathrm{~ms}$ were used for CID studies. The normalized collision energy was varied from $0 \%$ to $45 \%$ for the dissociation profile (breakdown) study. The maximum supplementary AC voltage applied to the end caps of the ion trap mass analyzer was $\sim 4 \mathrm{~V}$ (for $\mathrm{m} / z$ 500). Helium was used as the buffer gas in the collision cell (ion trap). Data acquisition was performed for 1 min using XCalibur software (Thermo Fisher Scientific).

\section{Results and Discussion}

\section{ESI-MS Study on Ni(II) Complex of 1,8-dimethyl- 1,3,6,8,10,13-hexaazacyclotetradecane (1)}

Synthesis and the solution chemistry of the Complex 1 were reported earlier by Suh et al. [23] They showed that the complex $[\mathrm{Ni}(\mathrm{L})]\left(\mathrm{ClO}_{4}\right)_{2} \quad(\mathrm{~L}=1,8$-dimethyl-1,3,6,8,10,13hexaazacyclotetradecane) is square-planar and is extremely stable in the crystalline state [24] as well as in solution. They also reported that [23] the metal ion $\left(\mathrm{Ni}^{2+}\right)$ was not released from Complex (1) even on treatment with strong demetalating agents such as $\mathrm{NaCN}, \mathrm{H}_{2} \mathrm{~S}$ gas, or strong acid, and Complex 1 showed 35\% decomposition on incubation for $10 \mathrm{~h}$ at $25^{\circ} \mathrm{C}$ in $0.3 \mathrm{M} \mathrm{HNO}_{3}(\mathrm{pH} \sim 0.52)$.

Since the Complex $\mathbf{1}$ is intrinsically di-positive and its molecular weight (without considering the perchlorate counter anion) is $\sim 288$, the expected molecular cation peak in the ESI-MS $\left(\mathrm{MS}^{1}\right)$ should appear at $m / z \sim 144$. But when we electrosprayed the aqueous solution of the Complex 1 in positive ion mode, the observed mass spectrum (Figure 2a) was complicated and consisted of total 10 peaks, including the peak at $\mathrm{m} / z 144.2$ (relative abundance $\sim 3 \%$ ). After close inspection of the spectrum and keeping apart the peak at $\mathrm{m} / \mathrm{z}$ 144.2 (see later), we grouped the remaining nine peaks into three sets (A0, B0, C0), (A1, B1, C1), and (A2, B2, C2) as shown in the Figure 2a. Two successive peaks in a set (i.e., $\mathrm{Ax}-\mathrm{Bx}$ and $\mathrm{Bx}-\mathrm{Cx}, \mathrm{x}=0,1,2)$ differ by $m / z 43 \pm 0.5$ and the same type of peak in two successive sets (i.e., Y0- Y1 and $\mathrm{Y} 1-\mathrm{Y} 2, \mathrm{Y}=\mathrm{A}, \mathrm{B}, \mathrm{C})$ are separated by $m / z 100 \pm 0.5$.

The observation of these peaks (Figure 2) cannot be interpreted by considering the ion pair formation of the Complex 1 with $\mathrm{ClO}_{4}{ }^{-}$or by the axial addition of water molecules to the square-planar Complex $\mathbf{1}$ converting it into the octahedral complex (report of Suh et al. showed that some of the square planar species are converted to the octahedral species in water $[23,26])$ and/or by protonation of the uncoordinated tertiary nitrogen atoms in the macrocyclic framework (Figure 1). The observation of the three sets of $\mathrm{A}, \mathrm{B}$, and $\mathrm{C}$ ions could thus only be rationalized by the consideration that the Complex 1 undergoes trans-

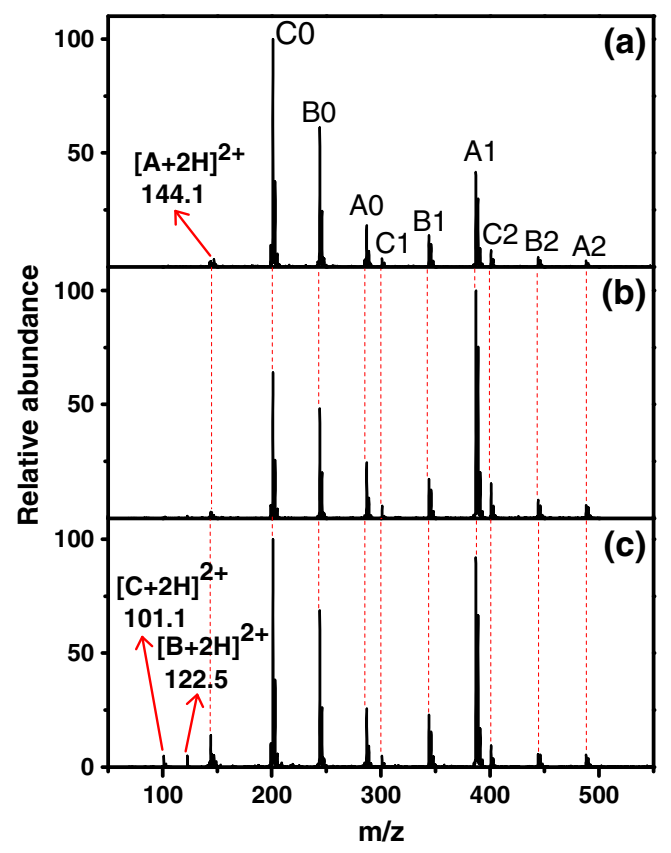

Figure 2. Representative mass spectra $\left(\mathrm{MS}^{1}\right)$ of $1(10 \mu \mathrm{M})$ electrosprayed from the solution of (a) water, (b) methanol, and (c) $1 \%$ (vol/vol) m-NBA in water 
formation/fragmentation along with ion pair formation with the $\mathrm{ClO}_{4}^{-}$anions. The $\mathrm{m} / \mathrm{z}$ difference of $100 \pm 0.5$ between the same type of peak in two successive sets supports the formation of ion pairs between $\mathrm{ClO}_{4}^{-}$(average molecular weight 99.5) and the protonated molecular fragment of Complex 1. Each observed peak in the spectrum (Figure 2a) showed characteristic isotopic distribution (Figure 1S in the Supporting Information) corresponding to the natural abundances [33] of nickel isotopes. The first two most abundant isotopes $\left({ }^{58} \mathrm{Ni}\right.$ : abundance $\sim 68 \%$ and ${ }^{60} \mathrm{Ni}$ : abundance $\sim 26 \%$ ) of nickel differ by $2 \mathrm{Da}$ in mass [33] (Table $1 \mathrm{~S}$ in the Supporting Information), which is equal to the separation between two most intense isotopic peaks in the mass spectrum (Figure 1S in the Supporting Information), support that these ions are indeed singly charged and the ions contain one nickel atom in the structure.

Several instrumental factors such as spray voltage, capillary temperature, capillary voltage and tube lens voltage etc. have earlier been shown to influence the analyte response in ESI-MS in certain systems [34]. The effects of these instrumental parameters were assessed by varying the capillary temperature from 100 to $300{ }^{\circ} \mathrm{C}$, capillary voltage from $-30 \mathrm{~V}$ to $30 \mathrm{~V}$ and tube lens offset from $-30 \mathrm{~V}$ to $30 \mathrm{~V}$ (see Figure 2S (a)-(c) in the Supporting Information). The results remained almost independent of these instrumental parameters, indicating absence of thermal dissociation [3537] in the heated capillary region or absence of fragmentation in the capillary-skimmer region (nozzle-skimmer dissociation [38]). Fragmentation of the Complex $\mathbf{1}$ in the corona discharge is unlikely in the positive ion mode as the electrical discharge takes place mainly in negative ion mode [32]. Moreover, no change of the spectral quality was observed when the sheath gas was changed to air (containing oxygen) instead of pure nitrogen, supporting that corona discharge $[3,32]$ does not affect the fate of the analyte in the present case.

Considering the structure of the Complex 1 and the results shown in Figure 2, a viable pathway for the possible molecular transformation and fragmentation of Complex $\mathbf{1}$ is proposed (Scheme 1). Scheme 1 shows that the first step involves simultaneous or consecutive loss of two protons from two secondary amine moieties coordinated to the nickel (II) to form a neutral species $\mathbf{A}$ (an inner metallic complex of first order [25]). Then A can successively release two neutral molecules (2-azaprop-1-ene; molecular weight 43) to produce species $\mathbf{B}$ and finally $\mathbf{C}$. The sequential protonation and ion-pair formation (Table 1) with $\mathrm{ClO}_{4}-$ of $\mathbf{A}, \mathbf{B}$, and $\mathbf{C}$ result in formation of singly positive molecular ions $\mathbf{A} n, \mathbf{B} n$, and $\mathbf{C} n(n=0,1,2)$ corresponding to peaks $\mathrm{A} n$, $\mathrm{B} n$, and $\mathrm{C} n,(n=0-2)$ depicted in the Figure 2a. Table 1 shows the $\mathrm{m} / \mathrm{z}$ values of the fragmented molecular ions and their $\mathrm{ClO}_{4}^{-}$adducts based on the Scheme 1 . The first set of peaks (A0, B0, C0) as discussed earlier thus actually corresponds to singly protonated molecular species of $\mathbf{A}$, $\mathbf{B}$, and $\mathbf{C}$. It is important to note that the singly charged species A0 may have the protonation either at one of the coordinated basic amine site (i.e., equivalent to the species formed by one proton loss from Complex 1) or at an uncoordinated amine site ( 1 or 8 positions) and these isomeric structures cannot be distinguished in the present experiment. The singly charged ion-pairs of multiply protonated $\mathbf{A}, \mathbf{B}$, and $\mathbf{C}$ with $\mathrm{ClO}_{4}^{-}$ions (molecular weight 95.5) give rise to the observation of the peaks $\mathbf{A} n, \mathbf{B} n$, and $\mathbf{C} n(n=1$ and 2) shown in the Figure 2a. The theoretical isotope profiles of the molecular ion species $\mathbf{A} n, \mathbf{B} n$ and $\mathbf{C} n$ $(n=0,1,2)$ calculated using Isopro 3.1 (http://sites.google. $\mathrm{com} / \mathrm{site} /$ isoproms/home) closely matched (Figure $3 \mathrm{~S}$ in the Supporting Information) with the experimentally observed isotopic distribution (Figure 1S in the Supporting Information) of the molecular ion species.

The oxidation state of the nickel $(+2)$ was assumed to remain unchanged during the molecular transformations of Complex 1 proposed in the Scheme 1. A close inspection of the experimental isotopic distribution of the molecular ion species $\mathbf{A} n, \mathbf{B} n$, and $\mathbf{C} n(n=0,1,2)$ (Figure $1 \mathrm{~S}$ in the Supporting Information) agrees with our assumption that the central nickel ion exists in +2 oxidation state. If nickel were in +3 oxidation state in the molecular species $\mathbf{A}, \mathbf{B}$, and $\mathbf{C}$, they would hold a net positive charge $(+1)$ and would appear at $\mathrm{m} / \mathrm{z} 286,243$, and 200 respectively, which were not observed. On the other hand, we observed ion signals at $\mathrm{m} / z$ 287, 244 and 201 (Figure 1S (g), (h), (i) in the Supporting Information), which actually correspond to the singly protonated molecular species A0, B0, and $\mathbf{C O}$ respectively (see Table 1) containing $\mathrm{Ni}(\mathrm{II})$ at the center.

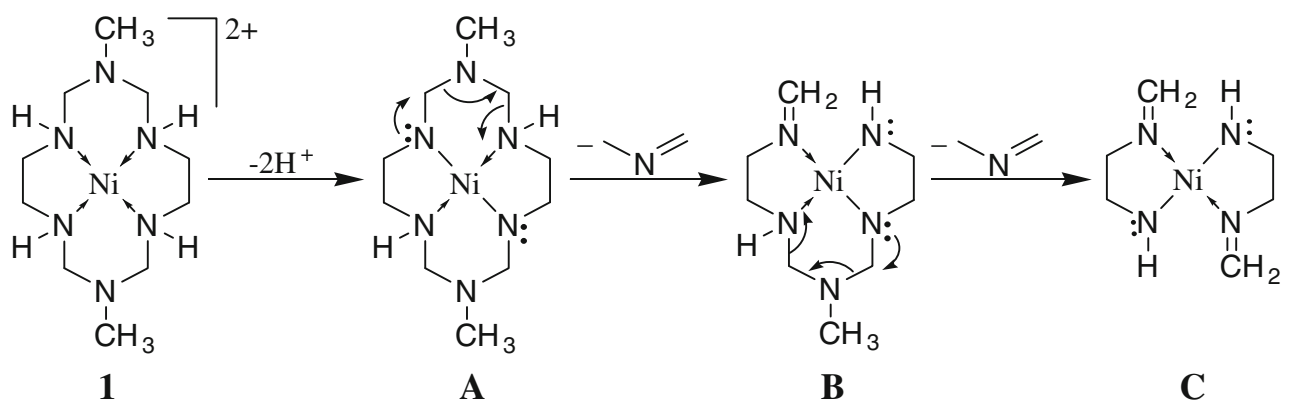

Scheme 1. The proposed fragmentation pathway of the Complex 1 
Table 1. The List of Molecular Ions Showing their Abbreviations Used in this Report and their Observed $m / z$ Values

\begin{tabular}{|c|c|c|c|c|c|c|}
\hline \multirow[t]{3}{*}{ Observed molecular ions } & \multicolumn{6}{|c|}{ Molecular fragments (M) } \\
\hline & \multicolumn{2}{|c|}{$\mathbf{A}$} & \multicolumn{2}{|c|}{ B } & \multicolumn{2}{|c|}{$\mathbf{C}$} \\
\hline & Abbreviation used ${ }^{\mathrm{a}}$ & Observed $m / z^{\mathrm{b}}$ & Abbreviation used ${ }^{\mathrm{a}}$ & Observed $m / z^{\mathrm{b}}$ & Abbreviation used ${ }^{\mathrm{a}}$ & Observed $m / z^{\mathrm{b}}$ \\
\hline$[\mathrm{M}+2 \mathrm{H}]^{2+}$ & - & $144.2 \pm 0.3$ & - & $122.4 \pm 0.3^{\mathrm{c}}$ & - & $101.1 \pm 0.3^{\mathrm{c}}$ \\
\hline$[\mathrm{M}+\mathrm{H}]^{+}$ & A0 & $287.0 \pm 0.3$ & B0 & $244.0 \pm 0.3$ & $\mathrm{CO}$ & $201.1 \pm 0.3$ \\
\hline$\left[\mathrm{M}+2 \mathrm{H}+\mathrm{ClO}_{4}\right]^{+}$ & A1 & $387.1 \pm 0.3$ & B1 & $344.1 \pm 0.3$ & $\mathrm{C} 1$ & $301.1 \pm 0.3$ \\
\hline$\left[\mathrm{M}+3 \mathrm{H}+2 \mathrm{ClO}_{4}\right]^{+}$ & A2 & $488.1 \pm 0.3$ & B2 & $444.1 \pm 0.3$ & $\mathrm{C2}$ & $401.1 \pm 0.3$ \\
\hline
\end{tabular}

${ }^{\text {a }}$ The abbreviation contains a capital letter representing the molecular fragment as shown in Scheme 1 and a digit representing the number of perchlorate ions forming the ion pair with the protonated molecular fragment ion.

${ }^{\mathrm{b}}$ Each peak observed in the mass spectra is basically a set of a number of isotopic peaks corresponding to the molecular ion. Here we report the $m / z$ of the most intense isotopic peak of each set with the standard error corresponding to the half width of the most intense isotopic peak taken into account. For convenience the nearest integer value of $\mathrm{m} / \mathrm{z}$ from the mean was considered in the discussion of this report.

${ }^{\mathrm{c}}$ Only observed in the mass spectrum of the complex 1 electrosprayed from $1 \% \mathrm{~m}$-NBA solution.

The observed deprotonation and fragmentation of the Complex 1 happen only during the electrospray ionization process in the positive ion mode as the Complex $\mathbf{1}$ is stable in the aspirating solution [23]. The positive ion mode of the electrospray ionization conventionally allows protonation of the analytes but not deprotonation, thus the proposed deprotonation phenomenon (Scheme 1) is not anticipated in conventional ESI process.

\section{Rationalization of the Deprotonation and Fragmentation of Complex 1}

In the electrospray process, the charged droplets are produced by the electrochemical reactions at the emitter surface [39]. This electrochemical reaction is assumed to be the oxidation of solvents [40] (in positive ion mode) producing protons in absence of any other redox active species in the solution $[32,40]$. Thus, in general, the main source of the excess positive charges produced in the droplet by ESI is the protons formed from the solvent electrolysis. The oxidation of the counter anion in the positive ion mode may be ruled out in the present case as the chlorine in $\mathrm{ClO}_{4}{ }^{-}$exists in its highest oxidation state (+7) [25]. The excess protons produced in the electrospray would lower the $\mathrm{pH}$ of the droplets. The lowest $\mathrm{pH}$ of the droplet estimated by considering the Rayleigh limit [41] of the positively charged droplet (see Supporting information) was $\sim 1.3$, which is much higher than the decomposition $\mathrm{pH}(\mathrm{pH} \sim 0.52)$ of Complex 1 reported earlier [23], and thus any $\mathrm{pH}$ induced decomposition of Complex $\mathbf{1}$ in the droplet under the present situation may not be possible.

The ion evaporation model (IEM) proposed by Fenn [19] suggested that the neutral analyte molecule undergoes Brownian motion inside the droplet leading it to encounter with the charged surface of the droplet. This may cause protonation of the analyte by association with the charge at the surface of the droplet. The thermal activation $(\mathrm{kT})$ and Coulomb repulsion may subsequently provide sufficient energy to desorb the protonated molecular species from the highly charged droplet surface. However, the ability of the formation of neutral ion-pair between Complex 1 with its counter ion $\left(\mathrm{ClO}_{4}^{-}\right)$would be very low in the present case because of the stable $\pi$-delocalization of the counter anion $\mathrm{ClO}_{4}^{-}$[25]. Moreover, the interaction between Complex $\mathbf{1}$ and its counter anion would be shielded in the aqueous solution owing to solvation of the ions in the high dielectric solvent. The $\mathrm{ClO}_{4}^{-}$ion might thus form neutral $\mathrm{HClO}_{4}$ inside the charged droplet by abstraction of a proton from the surface of the droplet. The positively charged Complex $\mathbf{1}$ may then be left without any counter ion for ion-pair formation and become destabilized inside the droplet due to Coulombic repulsion by the protons from the surface. This might lead to deprotonation of Complex $\mathbf{1}$ forming the neutral species (A), which is free from Coulomb repulsion, inside the core of the charged droplet. The neutral inner metallic Complex A would be distinctly different in structure and stability [25] compared with that of the charged noninner metallic Complex 1. The removal of protons from the secondary amine group might introduce strain in the sixmembered chelate ring of the metal complex, which causes the molecule $\mathbf{A}$ to undergo further fragmentation inside the charged droplet as depicted in the Scheme 1. The elimination of two neutral molecules (2-azaprop-1-ene) from the six-membered chelate rings of $\mathbf{A}$ would possibly remove the strain in the complex stabilizing the resulting neutral species $\mathbf{B}$ and $\mathbf{C}$ as has been shown in the Scheme 1. This scheme of reactions also agrees with the earlier reports $[23,26]$, which proposed that small changes in the bonding of the nitrogen atoms in the chelate rings of Complex 1 (e.g., replacement of the methyl groups by hydrogens in 1,8-position) could increase the strain in the chelate rings (Figure 1).

In order to assess the implications of the weak ion-pair forming ability of $\mathrm{ClO}_{4}^{-}$as discussed above, we carried out the experiments in presence of excess (100 folds) $\mathrm{NH}_{4} \mathrm{Cl}$ in the solution of Complex 1. Unlike the $\mathrm{ClO}_{4}^{-}$, the chloride ion makes tight neutral ion-pairs with the complex cation (1) inside the charged droplet that was clearly supported in the ESI-MS (Figure $4 \mathrm{~S}$ in the Supporting Information), which 
showed significantly reduced fragmentation efficiency of the Complex 1 in presence of $\mathrm{NH}_{4} \mathrm{Cl}$.

\section{Effect of Solvents on the Fragmentation of the Complex 1 During ESI-MS}

In order to investigate the role of the solvent on the nature of ESI-MS spectra, we electrosprayed the Complex 1 from different solvents like water, methanol and $1 \%$ (vol $/ \mathrm{vol}) \mathrm{m}$ NBA in water (Figure 2). We observed that the most intense peak (base peak) in the mass spectrum of Complex $\mathbf{1}$ in methanol was A1 while that in water was C0 (Figure 2b). As discussed above, the peak $\mathrm{C} 0$ corresponds to the protonated end product $\mathbf{C}(\mathbf{C 0}$; see Table 1, Scheme 1), but the peak A1 corresponds to the charged ion pair (A1; see Table 1). We calculated the fractional abundance of the individual ion-pair (A $n, \mathbf{B} n, \mathbf{C} n$ where $n=1,2$; see Table 1) over the total ion abundance from both spectra (Figure 2a and b) obtained by electrospray from the aqueous and from the alcoholic solution of Complex 1. The results shown in Table 2 indicate enhanced ion pair formation ability of all the fragments (A, B, and $\mathbf{C}$ ) when Complex $\mathbf{1}$ was electrosprayed from methanol solution instead of water because of low dielectric constant of the former solvent. These results thus support that the fragmentation indeed occurs inside the charged droplets. The protonated molecular fragments (the protonation is supposed to occur in the basic amine sites) $\mathbf{A}$, $\mathbf{B}$, and $\mathbf{C}$ may form ion-pairs with $\mathrm{ClO}_{4}-$ while ejecting from the charged surface of the droplet and the tendency for ion-pair formation was directly dependent on the dielectric constant of the solvent. The propensity of ion-pair formation of the fragment species (A, B, and $\mathbf{C}$ ) would not have been dependent on the dielectric constant of the solvent, if the fragmentation occurred outside the droplet (in gas phase) such as in the heated capillary and/or in the capillary skimmer region.

Figure 2a shows a peak at $\mathrm{m} / \mathrm{z} 144.2$ with $\sim 3 \%$ relative abundance in the ESI-MS of Complex 1 electrosprayed from water. As mentioned above, this peak could arise from the bare doubly charged cationic Complex 1 (di-positive and molecular weight 288). However, there is another possible ion (doubly protonated $\mathbf{A}$ ) formed from Complex $\mathbf{1}$ that may also contribute to this peak. The doubly protonated $\mathbf{A}$

Table 2. Percentage of the Total Ion Abundance for Different Charged Ion Pairs in the Mass Spectra $\left(\mathrm{MS}^{1}\right)$ of the Complex 1 Electrosprayed from Water and from Methanol

\begin{tabular}{crr}
\hline Charged ion pair & \multicolumn{2}{c}{ Percentage of the total ion abundance } \\
\cline { 2 - 3 } & Solvent: water & Solvent: methanol \\
\hline C1 & $1.00 \pm 0.05$ & $2.00 \pm 0.05$ \\
B1 & $6.00 \pm 0.05$ & $7.00 \pm 0.05$ \\
A1 & $17.00 \pm 0.05$ & $37.00 \pm 0.05$ \\
C2 & $2.00 \pm 0.05$ & $4.00 \pm 0.05$ \\
B2 & $1.00 \pm 0.05$ & $3.00 \pm 0.05$ \\
A2 & $1.00 \pm 0.05$ & $2.00 \pm 0.05$ \\
\hline
\end{tabular}

(Scheme 1) may be different from the original complex cation 1 as protonation could occur at the uncoordinated tertiary amine moieties at 1-and/or 8-positions in the neutral A.

We also examined the multiprotonation (supercharging) effect of the solvent on the analyte by addition of m-NBA (m-nitrobenzyl alcohol) to the aqueous electrospray solution [42]. Electrospray of Complex 1 from an aqueous solution containing $1 \% \mathrm{~m}-\mathrm{NBA}$ showed two additional peaks at $\mathrm{m} / \mathrm{z}$ 122.5 and $m / z 101.1$ (Figure 2c) corresponding to the doubly protonated $\mathbf{B}$ and $\mathbf{C}$, respectively, apart from all the peaks that were observed in the ESI-MS of Complex $\mathbf{1}$ in water (Figure 2a). Moreover, the relative abundance of the peak at $\mathrm{m} / \mathrm{z} 144.2$ that was suggested to have contributions from a doubly protonated form of $\mathbf{A}$ increases from $3 \%$ to $14 \%$ on addition of m-NBA. The Figure $2 \mathrm{c}$ also shows that the propensity of multiple charging of all the molecular fragments $(\mathbf{A}, \mathbf{B}$, and $\mathbf{C})$ increases in presence of $1 \% \mathrm{~m}-\mathrm{NBA}$ in the electrospray solution. If the fragmentation of Complex 1 (Scheme 1) had occurred in the gas-phase (i.e., outside the droplet) then the molecular fragments $\mathbf{A}, \mathbf{B}$, and $\mathbf{C}$ could not have sensed this multiple charging phenomenon leading to the formation of doubly protonated species in presence of $\mathrm{m}$ NBA in the aqueous solution. This result thus supports that all the fragments $\mathbf{A}, \mathbf{B}$, and $\mathbf{C}$ are produced inside the charged droplet.

\section{Collision-Induced Dissociation Experiments on the Ions Derived from the Complex 1}

The molecular fragments (A $n, \mathbf{B} n$, and $\mathbf{C} n$ where $\boldsymbol{n}=0,1,2)$ was further characterized by collision-induced dissociation (CID-MS/MS) experiments. All the ions observed in the $\mathrm{MS}^{1}$ spectrum of the Complex 1 (Figure 2a) were individually mass-selected and then allowed to undergo collisional activation with an inert gas (He) in the ion-trap (collision cell). Low energy collisional activation of the precursor ions (An, $\mathbf{B} n, \mathbf{C} n, \boldsymbol{n}=0-2)$ gave peaks (Figure $5 \mathrm{~S}$ in the Supporting Information) that were already observed in the $\mathrm{MS}^{1}$ of the Complex 1 (Figure 2a). The product ions formed by CID of the precursor ions are depicted in the MS ${ }^{1}$ spectrum by horizontal arrows between the precursor and the corresponding product ion peaks in Figure 3. The Figure 3 shows that if the precursor ion is $\mathbf{A} n(n=0,1,2$; see Table 1$)$ then it gives rise to the product ions $\mathbf{A}(n-\mathbf{1}), \mathbf{B} n$, and $\mathbf{C} n$. Similarly if the precursor ion is $\mathbf{B} n(n=0,1,2$; see Table 1$)$, then it produces the product ions $\mathbf{B}(n-\mathbf{1})$ or $\mathbf{C} n$ only. But if the precursor is $\mathbf{C} n(n=1,2$; see Table 1), then it only produce the $\mathbf{C}(n-\mathbf{1})$ ions. This result clearly suggests that $\mathbf{A}$ is the precursor of $\mathbf{B}$, and $\mathbf{B}$ is the precursor of $\mathbf{C}$, and thus supports the fragmentation of the Complex $\mathbf{1}$ proposed in Scheme 1.

In order to assess the relative stability of the singly protonated species (A0, B0, and C0), we performed energy resolved CID on them. Figure $6 \mathrm{~S}$ in the Supporting Information shows the dissociation profile of these molec- 


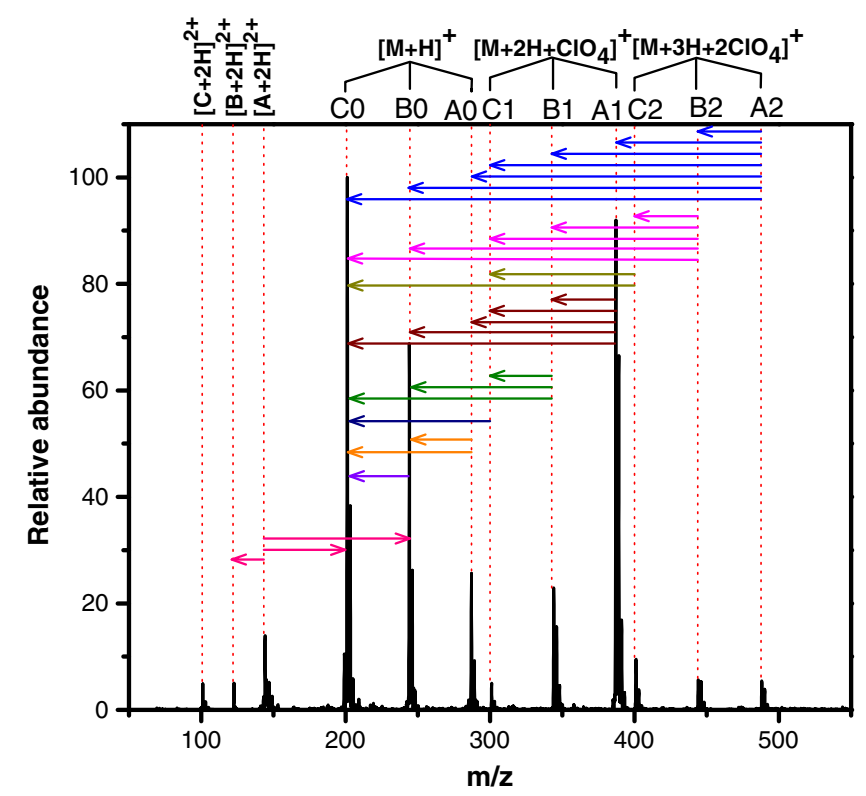

Figure 3. Mass spectrum $\left(\mathrm{MS}^{1}\right)$ of the Complex 1 electrosprayed from the solution of $1 \%$ (vol/vol) m-NBA in water (same as Figure 2c). CID-MS/MS on each ion (peak) produced the product ions that were also observed in $\mathrm{MS}^{1}$ spectrum. Each precursor ion peak is connected by particular color arrows to the corresponding product (fragment) ion peaks obtained from CID. The individual $\mathrm{MS}^{2}$ spectrum is shown in the Figure $5 S$ of the Supporting Information

ular ions at different collision energies. The breakdown curves (Figure 6S) for the singly protonated species show the stability order $\mathbf{A 0}<\mathbf{B 0}<\mathbf{C 0}$ suggesting that conversion of $\mathbf{A}$ to $\mathbf{B}$ and $\mathbf{C}$ by sequential release of 2-azaprop-1-ene as proposed in Scheme 1 is also energetically favorable.

Figure 3 shows that the $m / z 201.1$ (C) is the common peak in the $\mathrm{MS}^{2}$ of all mass-selected ions $\mathbf{A} n, \mathbf{B} n, \mathbf{C} n(n=0$ $2)$. In order to ascertain whether this peak indeed corresponds to one specific molecular ion (C0), as proposed in Scheme 1 or not, we performed $\mathrm{MS}^{3}$ of the $\mathrm{m} / \mathrm{z} 201.1$ (Figure 7S in the Supporting Information) derived from each $\mathrm{MS}^{2}$ spectrum (Figure $5 \mathrm{~S}$ in the Supporting Information). The $\mathrm{MS}^{3}$ spectra in all cases were identical to each other (data not shown). Moreover, the pattern of the $\mathrm{MS}^{3}$ spectra of the $\mathrm{m} / \mathrm{z}$ 201.1, matched with the $\mathrm{MS}^{2}$ spectra of $\mathbf{C 0}(\mathrm{m} / \mathrm{z}$ 201.1) shown in Figure 7S in the Supporting Information. This result suggests that the $\mathrm{m} / \mathrm{z} 201.1$ corresponds to the same ionic species $\mathbf{C O}$ as proposed in Scheme 1. The assignments of the peaks observed in the CID of $\mathrm{m} / \mathrm{z} 201.1$ are shown in Figure 6S in the Supporting Information and the possible fragmentation pathway of $\mathbf{C 0}$ based on the mobile proton model [43] is given in Scheme 1S.

The $\mathrm{m} / \mathrm{z} 144.2$ that originated from the dicationic Complex $\mathbf{1}$ and/or from the doubly protonated $\mathbf{A}$ was further investigated by CID. The molecular ion corresponding to the $\mathrm{m} / z 144.2$ was mass selected from ESI-MS of the Complex $\mathbf{1}$ in $1 \%$ aqueous m-NBA (Figure 2c) and CID was performed.
The results showed three product ions at $\mathrm{m} / \mathrm{z} 122.5, \mathrm{~m} / \mathrm{z}$ 201.1, and $\mathrm{m} / \mathrm{z} 244.0$ (Figure 5S (i) in the Supporting Information). Observation of $\mathrm{MS}^{2}$ fragment ions (product ions) having higher $\mathrm{m} / \mathrm{z}$ than the precursor ions $(\mathrm{m} / \mathrm{z}$ 144.2) confirms presence of doubly-protonated charge-state of the precursor ion.

\section{ESI-MS Study on 3,4,5:12,13,14-dipyridine- 2,6,11,15-tetramethyl-1,7,10,16-tetraallyl- 1,4, 7,10,13,16-hexaazacyclooctadeca-3,13-diene [macrocycle $\boldsymbol{M}_{4 p}$ ]}

We designed [27] the macrocycle $\mathbf{M}_{4 \mathbf{p}}$ with four allyl pendant arms (designated by subscript $4 p$ ) to the peripheral amines in the macrocyclic framework that may produce a resonance stabilized allyl cation $\left(\mathrm{CH}_{2}=\mathrm{CH}-\mathrm{CH}_{2}{ }^{+}\right)$on fragmentation at the protonated form in the electrospray droplet. Such a macrocycle should have strong proton affinity due to the presence of four tertiary amine groups in the periphery. Earlier work on similar pyridine containing amine macrocycle showed that protonation in acidic solution only occurs at the amine nitrogens, not at the pyridyl nitrogens [44].

It was interesting to note that the methanolic solution of $\mathbf{M}_{\mathbf{4 p}}$, showed multiple peaks in the ESI-mass spectrum $\left(\mathrm{MS}^{1}\right)$ as shown in the Figure 4a. Analogous to Complex 1, we also observed that the small variations of instrumental

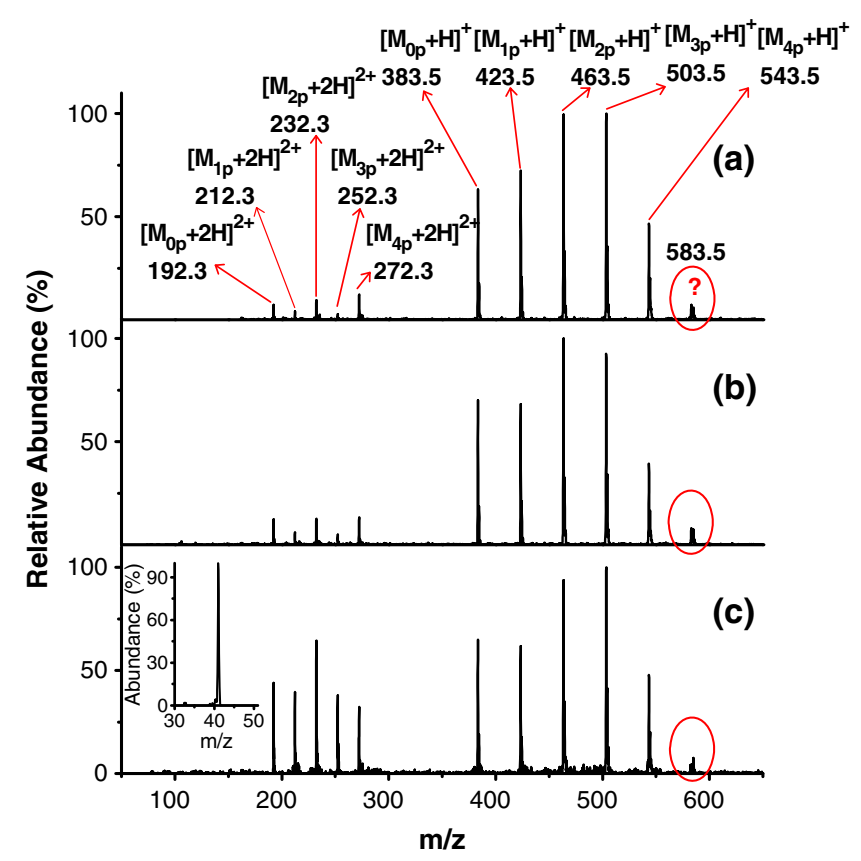

Figure 4. Representative mass spectra $\left(\mathrm{MS}^{1}\right)$ of the allyl pendant armed macrocycle $\mathbf{M}_{\mathbf{4 p}}(10 \mu \mathrm{M})$ electrosprayed from the solution of (a) methanol, (b) $5 \%$ (vol/vol) acetic acid in methanol and (c) $5 \%$ (vol/vol) m-NBA in methanol. Here the macrocycle containing ' $n$ ' number of allyl pendant arm is represented as $\mathbf{M}_{n}[n=4,3,2,1,0]$ 
parameters did not have any major effect on the mass spectrum of $\mathbf{M}_{4 \mathbf{p}}$. A close inspection of Figure 4a shows peaks at $\mathrm{m} / \mathrm{z} 543.5$ and $\mathrm{m} / \mathrm{z} 272.3$, respectively, for the mono- and di-protonation of the original macrocycle $\mathbf{M}_{4 \mathbf{p}}$. Figure $4 \mathrm{a}$ also shows peaks at $\mathrm{m} / \mathrm{z} 503.5,463.5,423.5$, and 383.5 , corresponding to singly charged species with successive $\mathrm{m} / \mathrm{z}$ difference of 40, and peaks at $\mathrm{m} / \mathrm{z} 252.3,232.3$, 212.3 , and 192.3 corresponding to doubly charged species with successive $\mathrm{m} / \mathrm{z}$ difference of 20 . These results indicate that the protonated macrocycle $\mathbf{M}_{\mathbf{4}}$ might undergo fragmentation reaction by sequentially releasing allyl cations $(\mathrm{m} /$ $z 41)$ in the electrospray process producing the macrocycles $\mathbf{M}_{3 \mathbf{p}}, \mathbf{M}_{\mathbf{2 p}}, \mathbf{M}_{\mathbf{1}}$, and $\mathbf{M}_{\mathbf{0 p}}$, respectively, with 3, 2, 1, and 0 pendent arms as shown in the Scheme 2.

The propensity of protonation of the amines in these macrocycles was found to depend on nature of the solvent. The ESI-mass spectra $\left(\mathrm{MS}^{1}\right)$ of the macrocycle $\mathbf{M}_{\mathbf{4 p}}$ from the solution of $5 \%(\mathrm{vol} / \mathrm{vol})$ acetic acid in methanol is shown in Figure $4 \mathrm{~b}$ and that from $5 \%$ (vol $/ \mathrm{vol}) \mathrm{m}-\mathrm{NBA}$ in methanol is shown in Figure 4c. Although the basic pattern of the spectra remains the same, the intensity ratios of diprotonated to monoprotonated molecular fragment ions (Int. $\left[\mathbf{M}_{\mathbf{n p}}+2 \mathrm{H}\right]^{2+} /$ Int. $\left.\left[\mathbf{M}_{\mathbf{n p}}+\mathrm{H}\right]^{+}, n=0-4\right)$ were found to increase in the following order: methanol $<5 \%$ acetic acid in methanol $<5 \%$ m-NBA in methanol. Earlier reports proposed that the ejection of the charged (protonated) analyte molecule from the electrospray droplet takes place from the least volatile component of the droplet [45]. Thus, methanol would be preferentially evaporated from the electrospray droplets [4]

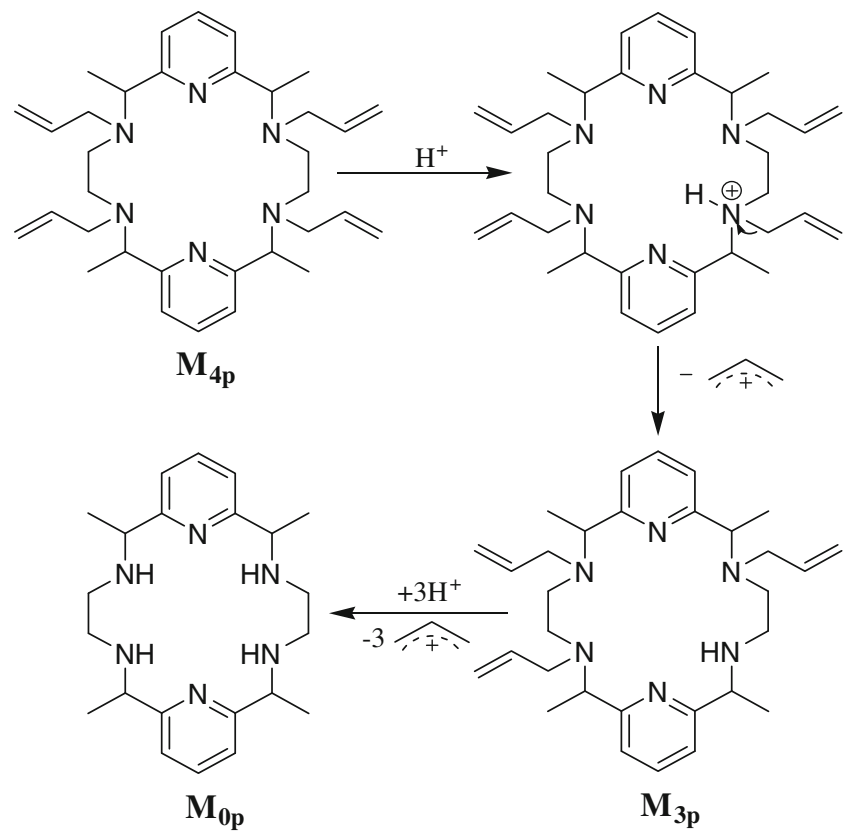

Scheme 2. The pathway of the successive acid cleavage of $\mathbf{M}_{\mathbf{4 p}}$ into the resonance stabilized allyl cations and $\mathbf{M}_{\mathbf{3 p}}, \mathbf{M}_{\mathbf{2 p}}$, $\mathbf{M}_{\mathbf{1 p}}, \mathbf{M}_{\mathbf{0 p}}$ in the electrospray process of the mixed solvents because of its higher vapor pressure compared to acetic acid or m-NBA (Table $2 \mathrm{~S}$ in the Supporting Information). This would eventually result in ejection of the analytes from the nano-droplets enriched with acetic acid or with m-NBA [45]. The surface tension of methanol $<$ acetic acid $<$ m-NBA (surface tension data are shown in Table $2 \mathrm{~S}$ in the Supporting Information). The charging of the analyte at the time of ejection would depend the number of charges $Z_{R}$, that can be sustained by a spherical droplet of radius, $R$, and surface tension, $\gamma$ given by the Rayleigh's equation (Equation 1) [41].

$$
Z_{R} . e=8 \pi\left(\epsilon_{0} \gamma R^{3}\right)^{1 / 2}
$$

Where $e$ is the elementary charge, and $\epsilon_{0}$ is the permittivity of the surrounding medium. So the droplet composed of liquid with high surface tension $(\gamma)$ would give rise to the enhanced charging in the gaseous analyte. If the fragmentation of the macrocycle $\mathbf{M}_{\mathbf{4}}$ had occurred in the gas-phase (i. e., outside the droplet) then we would not have observed the solvent dependence in the charging (protonation) of the fragmented molecules as shown in Table 3.

The maximum protonation sites of the macrocycle $\mathbf{M}_{\mathbf{4 p}}$ was earlier shown to be four [44]. Hence it can form a quadruply-protonated state $\left[\mathbf{M}_{\mathbf{4 p}}+4 \mathrm{H}\right]^{4+}$ along with singly-, doubly-, and triply-protonated states. If the protonated molecules were first desolvated from the electrospray droplets and subsequently fragmented (losing the allyl pendant arms) outside the droplet, the hypothetical gasphase fragmentation pattern would have followed Scheme 3. Scheme 3 shows that sequential removal of the allyl cation from the protonated $\mathbf{M}_{\mathbf{4}}$ can never lead to formation of protonated $\mathbf{M}_{\mathbf{0 p}}$, e.g., $\left[\mathbf{M}_{\mathbf{0 p}}+\mathrm{H}\right]^{+}$or $\left[\mathbf{M}_{\mathbf{0 p}}+2 \mathrm{H}\right]^{2+}$ and only the protonated forms of $\mathbf{M}_{\mathbf{1}}, \mathbf{M}_{\mathbf{2}}, \mathbf{M}_{\mathbf{3 p}}$ along with neutral $\mathbf{M}_{\mathbf{0 p}}$ would have formed. But the mass spectrum of $\mathbf{M}_{\mathbf{4 p}}$ clearly shows the peaks corresponding to the singly- and doubly-protonated $\mathbf{M}_{\mathbf{0 p}}$ at $m / z 192.3$ and 383.5, respectively (Figure 4), contrary to that expected from the Scheme 3. So this result supports that the fragmentation of $\mathbf{M}_{\mathbf{4 p}}$ indeed occurs inside the electrospray droplets.

Table 3. Intensity Ratio of Diprotonated to Monoprotonated Molecular Fragment Ions in the Mass Spectra $\left(\mathrm{MS}^{1}\right)$ of $\mathbf{M}_{\mathbf{4 P}}$ Electrosprayed from Different Solvents

\begin{tabular}{crcc}
\hline Molecular fragments & \multicolumn{3}{c}{$\begin{array}{l}\text { Percentage ratio of intensity of diprotonated } \\
\text { to that of monoprotonated species } \\
\left(I_{M+2 H^{2+}} / I_{M+H^{+}}\right) \times 100\end{array}$} \\
\cline { 2 - 4 } & $\begin{array}{l}\text { Solvent: } \\
\text { methanol }\end{array}$ & $\begin{array}{l}\text { Solvent: } 5 \% \text { acetic } \\
\text { acid (vol/vol) }\end{array}$ & $\begin{array}{l}\text { Solvent: } 5 \% \\
\text { m-NBA (vol/vol) }\end{array}$ \\
& $11.9 \pm 0.6$ & $17.0 \pm 0.9$ & $67.7 \pm 2.3$ \\
$\mathbf{M}_{\mathbf{0 p}}$ & $5.6 \pm 0.3$ & $8.7 \pm 0.4$ & $63.5 \pm 2.1$ \\
$\mathbf{M}_{\mathbf{1 p}}$ & $10.0 \pm 0.5$ & $12.5 \pm 0.6$ & $69.2 \pm 2.3$ \\
$\mathbf{M}_{\mathbf{2}}$ & $3.0 \pm 0.2$ & $5.4 \pm 0.3$ & $38.0 \pm 1.3$ \\
$\mathbf{M}_{\mathbf{3 p}}$ & $22.5 \pm 1.1$ & $33.3 \pm 1.7$ & $66.7 \pm 2.2$ \\
$\mathbf{M}_{\mathbf{4 p}}$ & \multicolumn{3}{c}{} \\
\hline
\end{tabular}




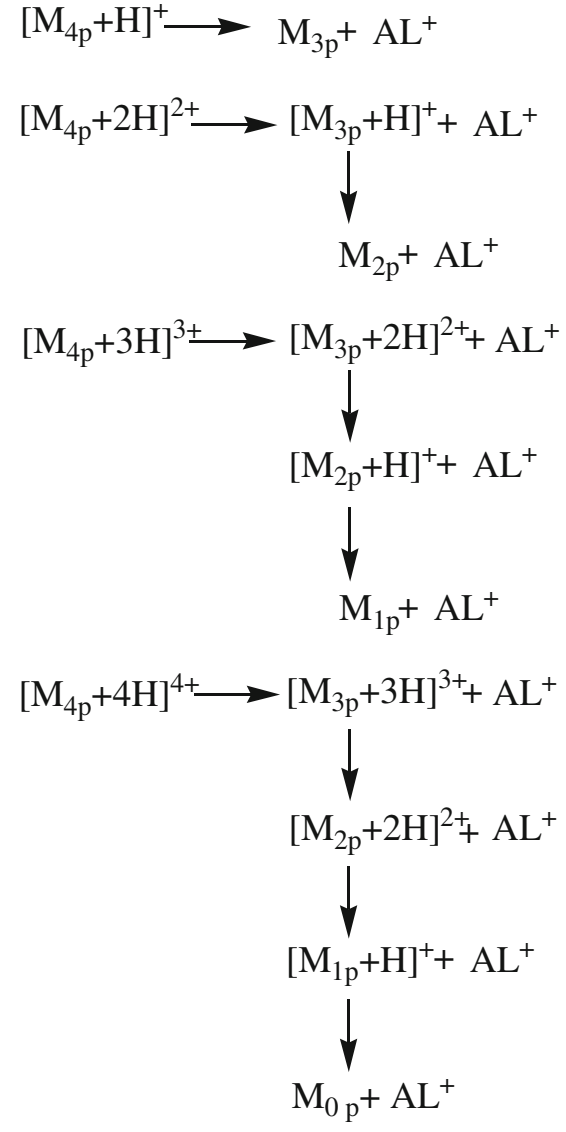

Scheme 3. The hypothetical gas-phase fragmentation reaction of the protonated macrocycle $\mathbf{M}_{\mathbf{4 p}}$ producing different charged as well as neutral macrocycle with different number of allyl pendant arms (p). $\mathrm{AL}^{+}$represents the allyl cation

The other evidence that the fragmentation of macrocycle $\mathbf{M}_{\mathbf{4 p}}$ occurs inside the charged droplets is the appearance of a weak ion signal at $\mathrm{m} / \mathrm{z} 583.5$ (Figure 4), which is higher than the $m / z$ of the precursor molecular ion $\left[\mathbf{M}_{\mathbf{4}}+\mathrm{H}\right]^{+}(\mathrm{m} / z$ 543.5). The following mechanism may be proposed in this case: the neutral analyte $\left(\mathbf{M}_{\mathbf{4 p}}\right)$ encounters the surface protons during Brownian dynamics inside the charged droplet [19], forming quaternary ammonium cation containing four allyl pendant arms $\left[\mathbf{M}_{\mathbf{4 p}}+\mathrm{nH}\right]^{\mathrm{n}+}(n=1-4)$. The Coulomb force of repulsion near the charged surface of the droplet would then lead the protonated $\mathbf{M}_{4 \mathbf{p}}$ to loss the resonance stabilized allyl cation as shown in the Scheme 2. Thus, the droplet surface becomes enriched with the allyl cations along with the protons. When the macrocycles are desorbed from the charged droplet surface they would have chances to abstract either protons or allyl cations by the amine groups present in the macrocyclic framework. The small peak at $\mathrm{m} / z 583.5$ suggests that $\mathbf{M}_{\mathbf{4 p}}$ has accommodated one more allyl cation (molecular weight 41) from the surface of the droplet during its desorption into the gas phase. Formation of the allyl cation $(\mathrm{m} / \mathrm{z} 41)$ as proposed in this mechanism was also detected at low mass range (see the inset of Figure 4c).

The allyl group has been frequently used in organic syntheses as a protecting group for amines due to its stability under both acidic and basic conditions [46]. Special synthetic efforts are required to deprotect these allyl protected amines. For example, Wilkinson's catalyst or a strong base $\left(\mathrm{KO}^{t} \mathrm{Bu}\right)$ causes the rearrangement of allyl amine to enamine, which then undergoes acid hydrolysis to liberate (deprotect) the amine [47-49]. Our results show that the electrospray ionization process may also act as an allyl scavenger to deprotect the amine group in the macrocycle $\mathbf{M}_{4 \mathbf{p}}$.

All these results provided compelling evidence to support that the environment around the analyte in the charged ES droplet can cause fragmentation of the analytes (Complex $\mathbf{1}$ and macrocycle $\mathbf{M}_{\mathbf{4 p}}$ ). It is important to note that the present experimental setup however cannot identify the stage of the electrospray ionization process at which the fragmentation reaction of the analyte is initiated. A charged droplet is formed from a Taylor cone (in the ES source), whose surface is also positively charged (under positive ion mode). So the fragmentation may also start at this zone and could continue in the droplet till the formation of the gas-phase ions. However, the Taylor cone is extremely short-lived, so the analyte would have too low of a residence time at this region to undergo significant fragmentation. Thus, most of the fragmentation processes possibly occur in the charged droplets.

\section{Conclusions}

The transformations of the analyte in the charged electrospray droplets have been studied by suitably choosing two analyte molecules viz. Ni(II) complex of 1,8-dimethyl1,3,6,8,10,13-hexaazacyclotetradecane (1) and allyl pendant armed macrocycle, 3,4,5:12,13,14-dipyridine-2,6,11,15-tetramethyl-1,7,10,16-tetraallyl-1,4,7,10,13,16-hexaazacyclooctadeca-3,13-diene $\left(\mathbf{M}_{\mathbf{4 p}}\right)$. Although we focused our study on the above two specific molecules, it provides an insight for the behavior of any electrosprayed molecule inside the charged droplet. Conventionally, electrospray ionization is considered as a soft-ionization technique, but the molecules may not always remain intact inside the electrospray droplet and they even may undergo transformations or fragmentations depending on their chemical nature and structure, as is evidenced in our present study. The tiny charged droplet produced by the electrospray process may thus be considered as a unique reaction vessel to induce a chemical reaction (charge induced chemical reaction) that may not be possible by conventional solution chemistry. The modifications and other transformations of proteins that are detected by ESI-MS may require a closer look in the future in the light of the results of the present studies. 


\section{Supporting Information}

Different isotopic abundances of nickel, physical properties of different electrospray solvents, ESI-MS ${ }^{1}$, simulated mass spectra and CID-MS/MS spectra of the different molecule/ fragment ions are shown in the Supporting Information.

\section{Acknowledgment}

The authors thank Mr. Bharat T. Kansara for his help. This work was supported by Tata Institute of Fundamental Research.

\section{References}

1. Fenn, J.B., Mann, M., Meng, C.K., Wong, S.F.: Whitehouse, C. M. Electrospray Ionization for Mass Spectrometry of Large Biomolecules. Science 246(4926), 64-71 (1989)

2. Yamashita, M., Fenn, J.B.: Electrospray Ion Source. Another Variation on the Free-Jet Theme. J. Phys. Chem. 88(20), 4451-4459 (1984)

3. Yamashita, M., Fenn, J. B.: Negative Ion Production with the Electrospray Ion Source. J. Phys. Chem. 88(20), 4671-4675 (1984)

4. Kebarle, P., Tang, L.: From Ions in Solution to Ions in the Gas Phasethe Mechanism of Electrospray Mass Spectrometry. Anal. Chem. 65 (22), 972A-986A (1993)

5. Banerjee, S., Mazumdar, S.: Noncovalent Dimers of the Lysine Containing Protonated Peptide Ions in Gaseous State: Electrospray Ionization Mass Spectrometric Study. J. Mass Spectrom. 45, 1212-1219 (2010)

6. Griffiths, W.J., Jonsson, A.P., Liu, S., Rai, D.K., Wang, Y.: Electrospray and Tandem Mass Spectrometry in Biochemistry. Biochem. J. 355 (3), 545-561 (2001)

7. Julian, R.R., Hodyss, R., Beauchamp, J.L.: Salt Bridge Stabilization of Charged Zwitterionic Arginine Aggregates in the Gas Phase. J. Am. Chem. Soc. 123(15), 3577-3583 (2001)

8. Wells, J.M., Chrisman, P.A., McLuckey, S.A.: Formation and Characterization of Protein-Protein Complexes in Vacuo. J. Am. Chem. Soc. 125(24), 7238-7249 (2003)

9. Daneshfar, R., Kitova, E.N., Klassen, J.S.: Determination of ProteinLigand Association Thermochemistry Using Variable-Temperature Nanoelectrospray Mass Spectrometry. J. Am. Chem. Soc. 126(15), 4786-4787 (2004)

10. Dole, M., Mack, L.L., Hines, R.L., Mobley, R.C., Ferguson, L.D., Alice, M.B.: Molecular Beams of Macroions. J. Chem. Phys. 49(5), 2240-2249 (1968)

11. Iribarne, J.V., Thomson, B.A.: On the Evaporation of Small Ions from Charged Droplets. J. Chem. Phys. 64(6), 2287-2294 (1976)

12. Thomson, B.A., Iribarne, J.V.: Field Induced Ion Evaporation from Liquid Surfaces at Atmospheric Pressure. J. Chem. Phys. 71(11), 44514463 (1979)

13. de la Mora, J.F.: Electrospray Ionization of Large Multiply Charged Species Proceeds Via Dole's Charged Residue Mechanism. Anal. Chim. Acta 406(1), 93-104 (2000)

14. Nemes, P., Marginean, I., Vertes, A.: Spraying Mode Effect on Droplet Formation and Ion Chemistry in Electrosprays. Anal. Chem. 79(8), 3105-3116 (2007)

15. Olumee, Z., Callahan, J.H., Vertes, A.: Droplet Dynamics Changes in Electrostatic Sprays of Methanol-Water Mixtures. J. Phy. Chem. A 102 (46), 9154-9160 (1998)

16. Richard, B. C. Some Tenets Pertaining to Electrospray Ionization Mass Spectrometry. J. Mass Spectrom. 35(7), 763-772 (2000)

17. Breuker, K., McLafferty, F.W.: Stepwise Evolution of Protein Native Structure with Electrospray into the Gas Phase, 10(-12) to 10(2) s. Proc. Natl. Acad. Sci. U.S.A. 105(47), 18145-18152 (2008)

18. Wood, T.D., Chorush, R.A., Wampler, F.M., Little, D.P., O'Connor, P.B., McLafferty, F.W.: Gas-Phase Folding and Unfolding of
Cytochrome c Cations. Proc. Natl. Acad. Sci. U.S.A. 92(7), 24512454 (1995)

19. Fenn, J.B.: Ion Formation from Charged Droplets: Roles of Geometry, Energy, and Time. J. Am. Soc. Mass Spectrom. 4(7), 524-535 (1993)

20. Ganan-Calvo, A.M.: The Surface Charge in Electrospraying: Its Nature and Its Universal Scaling Laws J. Aerosol Sci. 30(7), 863872 (1999)

21. Kaltashov, I.A., Mohimen, A.: Estimates of Protein Surface Areas in Solution by Electrospray Ionization Mass Spectrometry. Anal. Chem. 77 (16), 5370-5379 (2005)

22. Rodriguez-Cruz, S.E., Khoury, J.T., Parks, J.H.: Protein Fluorescence Measurements within Electrospray Droplets. J. Am. Soc. Mass Spectrom. 12(6), 716-725 (2001)

23. Suh, M.P., Kang, S.G. Synthesis and Properties of Nickel(II) and Copper(II) Complexes of 14-Membered Hexaaza Macrocycles, 1,8Dimethyl- and 1,8-Diethyl-1,3,6,8,10,13-Hexaazacyclotetradecane. Inorg. Chem. 27(14), 2544-2546 (1988)

24. Yan, Z., Li, T.: Crystal Structure of (1,8-Dimethyl-1,3,6,8,10,13Hexaazacyclotetradecane) Nickel(II) Diperchlorate, [Ni(C10H26N6)] [ClO4]2. Z. Kristallogr. NCS. 224, 601-602 (2009)

25. Sarkar, R. General and Inorganic Chemistry, Vol. 2. New Central Book Agency (P) Ltd., Kolkata (2001)

26. Suh, M.P., Shim, B.Y., Yoon, T.-S.: Template Syntheses and Crystal Structures of Nickel(II) Complexes of Hexaaza Macrocyclic Ligands with Pendant Functional Groups: Formation of a Coordination Polymer. Inorg. Chem. 33(24), 5509-5514 (1994)

27. Singh, A.K., Singh, P., Banerjee, S., Mehtab, S.: Development of Electrochemical Sensors for Nano Scale Tb(III) Ion Determination Based on Pendant Macrocyclic Ligands. Anal. Chim. Acta 633(1), 109118 (2009)

28. Prakash, H., Kansara, B.T., Mazumdar, S.: Effects of Salts on the Charge-State Distribution and the Structural Basis of the MostIntense Charge-State of the Gaseous Protein Ions Produced by Electrospray Ionization. Int. J. Mass Spectrom. 289(2/3), 84-91 (2010)

29. Prakash, H., Mazumdar, S.: Direct Correlation of the Crystal Structure of Proteins with the Maximum Positive and Negative Charge States of Gaseous Protein Ions Produced by Electrospray Ionization. J. Am. Soc. Mass Spectrom. 16(9), 1409-1421 (2005)

30. Prakash, H., Mazumdar, S.: Succinylation of Cytochrome $c$ Investigated by Electrospray Ionization Mass Spectrometry: Reactive Lysine Residues. Int. J. Mass Spectrom. 281(1/2), 55-62 (2009)

31. Hoffmann, E.d., Stroobant, V.: Mass Spectrometry Principles and Applications, 2nd ed. John Wiley and Sons, Ltd., Chichester (2001)

32. Richard, B.C.: Electrospray and MALDI Mass Spectrometry. John Wiley and Sons, New Jersey (2010)

33. Laeter, J.R.D., Bohlke, J.K., Bievre, P.D., Hidaka, H., Peiser, H.S., Rosman, K.J.R., Taylor, P.D.P.: Atomic Weights of the Elements. Review 2000 (IUPAC Technical Report). Pure Appl. Chem. 75(6), 683800 (2003)

34. Raji, M.A., Schug, K.A.: Chemometric Study of the Influence of Instrumental Parameters on ESI-MS Analyte Response Using Full Factorial Design. Int. J. Mass Spectrom. 279(2/3), 100-106 (2009)

35. Stipdonk, M.J.V., Ince, M.P., Perera, B.A., Martin, J.A.: Cluster Ions Derived from Sodium and Potassium Tetrafluoroborate and Their Collision Induced Dissociation in an Ion Trap Mass Spectrometer. Rapid Commun. Mass Spectrom. 16(5), 355-363 (2002)

36. Busman, M., Rockwood, A.L., Smith, R.D.: Activation Energies for Gas-Phase Dissociations of Multiply Charged Ions from Electrospray Ionization Mass Spectrometry. J. Phy. Chem. 96(6), 2397-2400 (1992)

37. Gabelica, V., De Pauw, E., Karas, M.: Influence of the Capillary Temperature and the Source Pressure on the Internal Energy Distribution of Electrosprayed Ions. Int. J. Mass Spectrom. 231(2/3), 189-195 (2004)

38. Yamada, N., Suzuki, E.-I., Hirayama, K.: Effective Novel Dissociation Methods for Intact Protein: Heat-Assisted Nozzle-Skimmer Collisionally Induced Dissociation and Infrared Multiphoton Dissociation Using a Fourier Transform Ion Cyclotron Resonance Mass Spectrometer 
Equipped with a Micrometal Electrospray Ionization emitter. Anal. Biochem. 348(1), 139-147 (2006)

39. Van Berkel, G.J.: Insights Into Analyte Electrolysis in an Electrospray Emitter from Chronopotentiometry Experiments and Mass Transport Calculations. J. Am. Soc. Mass Spectrom. 11(11), 951-960 (2000)

40. Van Berkel, G.J., Giles, G.E., Bullock; Gray, L.J.: Computational Simulation of Redox Reactions within a Metal Electrospray Emitter. Anal. Chem. 71(23), 5288-5296 (1999)

41. Rayleigh, L. Philos. Mag. 14, 184-186 (1882)

42. Iavarone, A.T., Jurchen, J.C., Williams, E.R.: Supercharged Protein and Peptide Ions Formed by Electrospray Ionization. Anal. Chem. 73(7), 1455-1460 (2001)

43. Boyd, R., Somogyi, Á.: The Mobile Proton Hypothesis in Fragmentation of Protonated Peptides: A Perspective. J. Am. Soc. Mass Spectrom. 21(8), 1275-1278 (2010)
44. Rothermel, G.L., Miao, L., Hill, A.L., Jackels, S.C.: Macrocyclic Ligands with 18-Membered Rings Containing Pyridine or Furan Groups: Preparation, Protonation, and Complexation by Metal Ions. Inorg. Chem. 31(23), 4854-4859 (1992)

45. Iavarone, A.T., Williams, E.R.: Mechanism of Charging and Supercharging Molecules in Electrospray Ionization. J. Am. Chem. Soc. 125 (8), 2319-2327 (2003)

46. Kocienski, P.J.: Protecting Groups, 3rd ed. Theime Verlag, Stuttgart (2003)

47. Corey, E.J., Suggs, J.W.: Selective Cleavage of Allyl Ethers under Mild Conditions by Transition Metal Reagents. J. Org. Chem. 38(18), 32243224 (1973)

48. Cunningham, J., Gigg, R., Warren, C.D.: The Allyl Ether as a Protection Group in Carbohydrate Chemistry. Tetrahedron Lett. 5(19), 1191-1196 (1964)

49. Gigg, J., Gigg, R.: The Allyl Ether as a Protecting Group in Carbohydrate Chemistry. J. Chem. Soc. C 82-86 (1966) 